\title{
Front Matter: Volume 7273
}

, "Front Matter: Volume 7273," Proc. SPIE 7273, Advances in Resist Materials and Processing Technology XXVI, 727301 (3 April 2009); doi:

10.1117/12.831120

SPIE. Event: SPIE Advanced Lithography, 2009, San Jose, California, United States 


\section{PROCEEDINGS OF SPIE}

\section{Advances in Resist Materials and Processing Technology XXVI}

\section{Clifford L. Henderson}

Editor

23-25 February 2009

San Jose, United States

Sponsored by

SPIE

Cooperating Organization

SEMATECH Inc. (United States)

Published by

SPIE 
The papers included in this volume were part of the technical conference cited on the cover and title page. Papers were selected and subject to review by the editors and conference program committee. Some conference presentations may not be available for publication. The papers published in these proceedings reflect the work and thoughts of the authors and are published herein as submitted. The publisher is not responsible for the validity of the information or for any outcomes resulting from reliance thereon.

Please use the following format to cite material from this book:

Author(s), "Title of Paper," in Advances in Resist Materials and Processing Technology XXVI, edited by Clifford L. Henderson, Proceedings of SPIE Vol. 7273 (SPIE, Bellingham, WA, 2009) Article CID Number.

ISSN 0277-786X

ISBN 9780819475268

Published by

SPIE

P.O. Box 10, Bellingham, Washington $98227-0010$ USA

Telephone +1 3606763290 (Pacific Time) · Fax +1 3606471445

SPIE.org

Copyright (c) 2009, Society of Photo-Optical Instrumentation Engineers

Copying of material in this book for internal or personal use, or for the internal or personal use of specific clients, beyond the fair use provisions granted by the U.S. Copyright Law is authorized by SPIE subject to payment of copying fees. The Transactional Reporting Service base fee for this volume is $\$ 18.00$ per article (or portion thereof), which should be paid directly to the Copyright Clearance Center (CCC), 222 Rosewood Drive, Danvers, MA 01923. Payment may also be made electronically through CCC Online at copyright.com. Other copying for republication, resale, advertising or promotion, or any form of systematic or multiple reproduction of any material in this book is prohibited except with permission in writing from the publisher. The CCC fee code is 0277-786X/09/\$18.00.

Printed in the United States of America.

Publication of record for individual papers is online in the SPIE Digital Library.

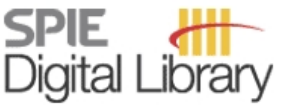

SPIEDigitalLibrary.org

Paper Numbering: Proceedings of SPIE follow an e-First publication model, with papers published first online and then in print and on CD-ROM. Papers are published as they are submitted and meet publication criteria. A unique, consistent, permanent citation identifier (CID) number is assigned to each article at the time of the first publication. Utilization of CIDs allows articles to be fully citable as soon they are published online, and connects the same identifier to all online, print, and electronic versions of the publication. SPIE uses a six-digit CID article numbering system in which:

- The first four digits correspond to the SPIE volume number.

- The last two digits indicate publication order within the volume using a Base 36 numbering system employing both numerals and letters. These two-number sets start with 00, 01, 02, 03, 04 , 05, 06, 07, 08, 09, OA, OB ... 0Z, followed by 10-1Z, 20-2Z, etc.

The CID number appears on each page of the manuscript. The complete citation is used on the first page, and an abbreviated version on subsequent pages. Numbers in the index correspond to the last two digits of the six-digit CID number. 


\section{Contents}

xvii Symposium Committee

xix Conference Committee

\section{DOUBLE PATTERNING AND DOUBLE EXPOSURE I}

$727304 \quad$ Newly developed positive tone resists for Posi/Posi double patterning process [7273-03] T. Nakamura, M. Takeshita, S. Maemori, Tokyo Ohka Kogyo Co., Ltd. (Japan); R. Uchida, Tokyo Ohka Kogyo America, Inc. (United States); R. Takasu, K. Ohmori, Tokyo Ohka Kogyo Co., Ltd. (Japan)

727305 Engine for characterization of defects, overlay and critical dimension control for double exposure processes for advanced logic nodes [7273-179]

S. Holmes, C. Koay, K. Petrillo, K.-J. Chen, M. E. Colburn, IBM Corp. (United States);

J. Cantone, K. Ueda, Tokyo Electron America, Inc. (United States); A. Metz, S. Dunn, Tokyo Electron Technology Ctr., America, LLC (United States); Y. van Dommelen, M. Crouse, J. Galloway, E. Schmitt-Weaver, A. Jiang, R. Routh, ASML (United States); C. Tang, M. Slezak, JSR Micro, Inc. (United States); S. Kini, T. DiBiase, KLA-Tencor New York (United States)

727306 Materials for single-etch double patterning process: surface curing agent and thermal cure resist [7273-05]

Y. C. Bae, Y. Liu, T. Cardolaccia, J. C. McDermott, P. Trefonas, K. Spizuoco, M. Reilly, A. Pikon, L. Joesten, G. G. Zhang, G. G. Barclay, Rohm and Haas Electronic Materials (United States);

J. Simon, S. Gaurigan, Commissariat à I'Energie Atomique (France)

727307 Double-exposure materials for pitch division with 193nm lithography: requirements, results [7273-06]

R. Bristol, D. Shykind, S. Kim, Y. Borodovsky, E. Schwartz, C. Turner, Intel Corp. (United States); G. Masson, K. Min, K. Esswein, J. M. Blackwell, Intel Corp., LBNL Molecular Foundry (United States); N. Suetin, Intel Corp. (Russian Federation)

727308 Topcoat-free ArF negative tone resist [7273-07]

T. Ando, S. Abe, R. Takasu, J. Iwashita, S. Matsumaru, R. Watanabe, K. Hirahara, Y. Suzuki, M. Tsukano, T. Iwai, Tokyo Ohka Kogyo Co., Ltd. (Japan)

\section{DOUBLE PATTERNING AND DOUBLE EXPOSURE II}

727309 Correlation of experimental and simulated cure-induced photoresist distortions in double patterning [7273-08]

T. I. Wallow, M. Rayasam, GlobalFoundries (United States); M. Yamaguchi, Y. Yamada, Ushio Inc. (Japan); K. Petrillo, IBM Corp. (United States); K. Yoshimoto, J. Kye, R. Kim,

H. J. Levinson, GlobalFoundries (United States) 
7273 OA Fine trench patterns with double patterning and trench shrink technology [7273-09] S. Shimura, Tokyo Electron Kyushu Ltd. (Japan); M. Kushibiki, Tokyo Electron AT Ltd. (Japan); T. Kawasaki, Tokyo Electron Kyushu Ltd. (Japan); R. Tanaka, A. Tokui, Y. Ishii, Nikon Corp. (Japan)

7273 OB Double patterning process with freezing technique [7273-10]

G. Wakamatsu, Y. Anno, M. Hori, T. Kakizawa, M. Mita, K. Hoshiko, T. Shioya, K. Fujiwara,

S. Kusumoto, Y. Yamaguchi, T. Shimokawa, JSR Corp. (Japan)

7273 OC Development of materials and processes for negative tone development toward 32-nm node 193-nm immersion double-patterning process [7273-11]

S. Tarutani, T. Hideaki, S. Kamimura, FUJIFILM Corp. (Japan)

7273 OD Photoresist stabilization for double patterning using $172 \mathbf{n m}$ photoresist curing [7273-12]

T. I. Wallow, GlobalFoundries (United States); J. Dai, Sokudo Co., Ltd. (United States);

C. R. Szmanda, Rohm and Haas Electronic Materials (United States); H. Cervera, Sokudo Co., Ltd. (United States); C. Truong, Rohm and Haas Electronic Materials (United States);

N. Bekiaris, Sokudo Co., Ltd. (United States); J.-W. Kye, R.-H. Kim, H. J. Levinson,

GlobalFoundries (United States); G. Mori, Sokudo Co., Ltd. (United States)

POSTER SESSION: NOVEL MATERIALS, PROCESSES, AND APPLICATIONS

7273 OE DUV-assisted e-beam resist process [7273-59]

W.-S. Chen, Industrial Technology Research Institute (Taiwan); Y.-C. Li, Everlight Chemical Industrial Corp. (Taiwan); M.-J. Tsai, Industrial Technology Research Institute (Taiwan)

7273 OF Improved CD uniformity for chemical shrink patterning [7273-60]

L. Chen, N. Bekiaris, T. Michaelson, G. Mori, Sokudo Co., Ltd (United States)

7273 OG Fabrication of 22-nm poly-silicon gate using resist shrink technology [7273-61]

F. Iwao, S. Shimura, T. Kawasaki, Tokyo Electron Kyushu Ltd. (Japan); M. Kushibiki, N. Eiichi, Tokyo Electron AT Ltd. (Japan)

$7273 \mathrm{OH} \quad$ Properties of the novel deprotecting unit for next-generation ArF resist polymer [7273-63] A. Shibuya, S. Yamaguchi, Y. Yoshida, M. Shirakawa, FUJIFILM Corp. (Japan)

7273 0J Chemically amplified hybrid resist plafform for i-line applications [7273-65] M. Toukhy, M. Paunescu, Z. Bogusz, G. Pawlowski, AZ Electronic Materials USA Corp. (United States)

7273 OK Criteria for success in e-beam resists [7273-66]

A. E. Zweber, IBM Corp. (United States); T. Komizo, Toppan Photomasks, Inc. (United States); J. Levin, Z. Benes, IBM Corp. (United States)

$7273 \mathrm{OL} \quad 0.30 \mathrm{k} 1 \mathrm{CH}$ delineation with novel image reversal materials [7273-67]

J. Hatakeyama, K. Katayama, T. Yoshihara, Y. Kawai, T. Ishihara, Shin-Etsu Chemical Co., Ltd. (Japan)

$72730 \mathrm{M} \quad$ KrF resists and process for implant layers at advanced nodes [7273-68]

H.-C. Huang, Y.-F. Huang, S. Wu, L. Jang, S. S. Lee, G. K. C. Huang, H. Chen, C. C. Yu, United Microelectronics Corp. (Taiwan); T. Kurihara, H. Fukiya, H. Yoshida, Y. Yamamoto, Rohm and Haas Electronic Materials (United States) 
7273 ON Process-induced bias: a study of resist design, device node, illumination conditions, and process implications [7273-69]

M. Carcasi, S. Scheer, C. Fonseca, Tokyo Electron America, Inc. (United States); T. Shibata, H. Kosugi, Y. Kondo, T. Saito, Tokyo Electron Kyushu Ltd. (Japan)

727300 Microbridge and e-test opens defectivity reduction via improved filtration of photolithography fluids [7273-70]

M. Mesawich, M. Sevegney, B. Gotlinsky, Pall Corp. (United States); S. Reyes, P. Abbott, J. Marzani, M. A. Rivera, Freescale Semiconductor, Inc. (United States)

7273 OP Post-develop blob defect reduction [7273-71]

M. Harumoto, S. Negoro, A. Hisai, SOKUDO Co., Ltd. (Japan); M. Tanaka, G. Mori, SOKUDO Co., Ltd. (United States); M. Slezak, JSR Micro, Inc. (United States)

7273 OR Embedded micro/nano channel formation for three-dimensional negative-tone photoresist microstructuring [7273-73]

S.-K. Kim, H.-K. Oh, Y.-D. Jung, I. An, Hanyang Univ. (Korea, Republic of)

7273 OS Analysis of the effect of point-of-use filtration on microbridging defectivity [7273-75] J. Braggin, IMEC vzW (Belgium) and Entegris, Inc. (United States); R. Gronheid, S. Cheng, D. Van Den Heuvel, S. Bernard, P. Foubert, IMEC vzW (Belgium); C. Rosslee, IMEC vzW (Belgium) and SOKUDO Co., Ltd. (United States)

7273 OT Novel resist for replica preparation of mold for imprint lithography [7273-77]

D. Matsukawa, H. Wakayama, K. Mitsukura, H. Okamura, Y. Hirai, M. Shirai, Osaka Prefecture Univ. (Japan)

7273 OU Pressure control for reduced microbubble formation [7273-78]

J. Braggin, Entegris, Inc. (United States)

7273 OV Productivity improvement in the wafer backside cleaning before exposure [7273-79] S. Nishikido, T. Kitano, Y. Tokunaga, Tokyo Electron Kyushu Ltd. (Japan); M. Strobl, Y. C. Lin, Inotera Memories, Inc. (Taiwan)

7273 OW Development of novel UV cross-linkable materials for enhancing planarity in via applications via the correlation of simulated and experimental analyses [7273-80] S. Takei, Nissan Chemical Industries, Ltd. (Japan); M. W. Lin, The Univ. of Texas at Austin (United States); S. Yoon, T. Ohashi, Y. Nakajima, Nissan Chemical Industries, Ltd. (Japan); C. G. Willson, The Univ. of Texas at Austin (United States)

7273 OX Comparison of thermal flow and chemical shrink processes for $193 \mathrm{~nm}$ contact hole patterning [7273-82]

T. Kudo, C. Antonio, J. Sagan, S. Chakrapani, D. Parthasarathy, S. Hong, M. Thiyagarajan,

Y. Cao, M. Padmanaban, AZ Electronic Materials USA Corp. (United States)

POSTER SESSION: ARCS AND MULTILAYER PROCESSES

7273 OY Message to the undecided: using DUV dBARC for 32-nm node implants [7273-25]

H.-R. Lee, SAMSUNG Electronics Co., Ltd. (United States); I. Y. Popova, J. M. Rolick, IBM

Microelectronics (United States); J.-M. Gomez, Freescale Semiconductor, Inc.

(United States); T. C. Bailey, IBM Microelectronics (United States) 
$7273 \mathrm{OZ}$ Investigation of the foot-exposure impact in hyper-NA immersion lithography when using thin anti-reflective coating [7273-84]

D. Jurajda, Brewer Science, Inc. (United States); E. Tenaglia, Numonyx Srl (Italy);

J. Jeauneau, Brewer Science, Inc. (United States); D. De Simone, Numonyx Srl (Italy); Z. Zhu,

Brewer Science, Inc. (United States); P. Piazza, P. Piacentini, P. Canestrari, Numonyx Srl (Italy)

727310 Organic underlayer materials with exceptionally high thermal stability [7273-85]

H.-S. Cheon, K.-H. Yoon, M.-S. Kim, S. B. Oh, J. Y. Song, N. Tokareva, J. S. Kim, T. Chang,

Samsung Cheil Industries Inc. (Korea, Republic of)

727311 Effects of carbon/hardmask interactions on hardmask performance [7273-86]

C. J. Neef, B. Smith, C. James, Z. Zhu, M. Weigand, Brewer Science, Inc. (United States)

727312 Improving the performance of light-sensitive developer-soluble anti-reflective coatings by using adamantyl terpolymers [7273-87]

J. D. Meador, J. A. Lowes, C. Stroud, S. Thomas, Y. Qiu, R.-M. L. Mercado, Brewer Science,

Inc. (United States); V. Pham, M. Slezak, JSR Micro, Inc. (United States)

727313 Controlling etch properties of silicon-based antireflective spin-on hardmask materials [7273-88]

S. K. Kim, H. M. Cho, C. Woo, S. R. Koh, M. Kim, H. C. Yoon, W. Lee, S.-W. Shin, J. S. Kim,

T. Chang, Samsung Cheil Industries Inc. (Korea, Republic of)

727316 Radiation sensitive developable bottom anti-reflective coatings (DBARC): recent results [7273-91]

F. M. Houlihan, A. Dioses, T. Kudo, M. Li, L. Zhang, S. Vasanthan, S. Chakrapani,

D. Parthasarathy, C. Antonio, E. Ng, P.-H. Lu, M. Neisser, M. Padmanaban, AZ Electronic

Materials (United States)

\section{POSTER SESSION: DOUBLE PATTERNING AND DOUBLE EXPOSURE}

7273 1A Utilization of spin-on and reactive ion etch critical dimension shrink with double patterning for $32 \mathrm{~nm}$ and beyond contact level interconnects [7273-96]

K. Petrillo, D. Horak, S. Fan, E. McLellan, M. Colburn, IBM Corp. (United States); A. Metz, S. Dunn, D. Hetzer, Tokyo Electron Technology Ctr. (United States); J. Cantone, K. Ueda, T. Winter, Tokyo Electron America, Inc. (United States); V. Balasubramaniam, Tokyo Electron Technology Ctr. (United States); C. Tang, M. Slezak, JSR Micro, Inc. (United States)

7273 1B Optical threshold layer and intermediate state two-photon PAG approaches to double exposure lithography [7273-97]

A. J. Berro, X. Gu, The Univ. of Texas at Austin (United States); N. O'Connor, S. Jockusch, Columbia Univ. (United States); T. Nagai, T. Ogata, The Univ. of Texas at Austin (United States); P. Zimmerman, B. J. Rice, SEMATECH Inc. (United States); E. Adolph, T. Byargeon, J. Gonzalez, The Univ. of Texas at Austin (United States); N. J. Turro, Columbia Univ. (United States); C. G. Willson, The Univ. of Texas at Austin (United States)

7273 1C Fundamental study of optical threshold layer approach towards double exposure lithography [7273-98]

X. Gu, A. J. Berro, Y. Cho, K. Jen, S. Lee, T. Ngai, T. Ogata, W. J. Durand, The Univ. of Texas at Austin (United States); A. Sundaresan, J. R. Lancaster, S. Jockusch, Columbia Univ. (United States); P. Zimmerman, Intel Corp. assignee to SEMATECH Inc. (United States); N. J. Turro, Columbia Univ. (United States); C. G. Willson, The Univ. of Texas at Austin (United States) 
7273 ID CD uniformity improvement for double-patterning lithography (litho-litho-etch) using freezing process [7273-101]

H. Sugimachi, H. Kosugi, T. Shibata, J. Kitano, Tokyo Electron Kyushu Ltd. (Japan); K. Fujiwara, K. Itou, M. Mita, A. Soyano, S. Kusumoto, M. Shima, Y. Yamaguchi, JSR Corp. (Japan)

$7273 \mathrm{IE} \quad$ Contact formation with extremely low proximity effect by double patterning technology [7273-102]

C. W. Yeh, S. S. Yu, H. J. Lee, C. H. Huang, E. Yang, T. H. Yang, K. C. Chen, C.-Y. Lu, Macronix International Co., Ltd. (Taiwan)

$7273 \mathrm{IF} \quad$ Double imaging with resist freezing in a vapor reaction chamber [7273-104]

R. R. Dammel, Y. Takano, R. Collett, D. J. Abdallah, AZ Electronic Materials (United States)

$72731 \mathrm{G}$ Study of the simulation parameter for EUVL [7273-105]

A. Sekiguchi, Litho Tech Japan Co., Ltd. (Japan)

7273 1H Process latitude simulation of positive-tone litho-litho-etch double patterning [7273-106]

W. P. Printz, S. A. Scheer, Tokyo Electron America, Inc. (United States)

$727311 \quad$ Performance of an ArF siloxane BARC exposed to a 172-nm UV cure for double patterning applications [7273-107]

Z.-Y. Wu, J. Kennedy, S.-Y. Xie, R. Katsanes, K. Flanigan, Honeywell Electronic Materials (United States); J. Dai, N. Bekiaris, H. Cervera, G. Mori, SOKUDO Co., Ltd. (United States);

T. Wallow, Advanced Micro Devices, Inc./The Foundry Co. (United States)

\section{POSTER SESSION: EUV RESIST MATERIALS AND PROCESSES}

$72731 \mathrm{~J} \quad$ Underlayer designs to enhance the performance of EUV resists [7273-109]

H. XU, Brewer Science, Inc. (United States); J. M. Blackwell, Intel Corp., LBNL Molecular Foundry (United States); T. R. Younkin, Intel Corp. (United States); K. Min, Intel Corp., LBNL Molecular Foundry (United States)

7273 IK Resolution and LWR improvements by acid diffusion control in EUV lithography [7273-108] H. Tsubaki, T. Tsuchihashi, T. Tsuchimura, FUJIFILM Co., Ltd. (Japan)

7273 IL EUV lithography for 30nm half pitch and beyond: exploring resolution, sensitivity, and LWR tradeoffs [7273-110]

E. S. Putna, T. R. Younkin, M. Chandhok, K. Frasure, Intel Corp. (United States)

$72731 \mathrm{M}$ Development of EUV resists at Selete [7273-111]

H. Oizumi, D. Kawamura, K. Kaneyama, S. Kobayashi, T. Itani, Semiconductor Leading Edge Technologies, Inc. (Japan)

7273 IN Non-chemically amplified negative resist for EUV lithography [7273-112]

M. Shirai, K. Maki, H. Okamura, Osaka Prefecture Univ. (Japan); K. Kanayama, T. Itani, Semiconductor Leading Edge Technologies, Inc. (Japan)

727310 Pattern-transfer process development for EUVL [7273-113]

D. Kawamura, Y. Tanaka, T. Itani, E. Soda, N. Oda, Semiconductor Leading Edge Technologies, Inc. (Japan) 
7273 IP EUV resist outgassing quantification and application [7273-114]

S. Kobayashi, J. J. Santillan, H. Oizumi, T. Itani, Semiconductor Leading Edge Technologies, Inc. (Japan)

$72731 Q \quad$ EUV resist processing in vacuum [7273-115]

K. Kaneyama, S. Kobayashi, T. Itani, Semiconductor Leading Edge Technologies, Inc. (Japan)

7273 IR Non-ionic PAG behavior under high energy exposure sources [7273-116]

R. A. Lawson, D. E. Noga, L. M. Tolbert, C. L. Henderson, Georgia Institute of Technology (United States)

7273 IW Sensitivity of EUV resists to out-of-band radiation [7273-121]

J. M. Roberts, R. L. Bristol, T. R. Younkin, Intel Corp. (United States); T. H. Fedynyshyn,

D. K. Astolfi, A. Cabral, MIT Lincoln Lab. (United States)

7273 1X Dissolution kinetics and deprotection reaction in chemically amplified resists upon exposure to extreme ultraviolet radiation [7273-122]

H. Yamamoto, T. Kozawa, S. Tagawa, Osaka Univ. (Japan); T. Mimura, T. Iwai, J. Onodera, Tokyo Ohka Kogyo Co., Ltd. (Japan)

7273 IY Evaluation of alcoholic hydroxyl derivatives for chemically amplified extreme ultraviolet resist [7273-123]

K. Furukawa, Mitsubishi Gas Chemical Co., Inc. (Japan); T. Kozawa, S. Tagawa, Osaka Univ. (Japan)

$727312 \quad$ Evaluation of novel resist materials for EUV lithography [7273-124]

I. Aratani, S. Matsunaga, T. Kajiyashiki, Kuraray Co., Ltd. (Japan); T. Watanabe, H. Kinoshita, Univ. of Hyogo (Japan)

727320 Evaluation of track performance for EUV lithography [7273-125]

K. Tanaka, Tokyo Electron Kyushu Ltd. (Japan) and Semiconductor Leading Edge Technologies, Inc. (Japan); J. Nakamura, Y. Yamada, Tokyo Electron Kyushu Ltd. (Japan);

S. Kobayashi, T. Itani, Semiconductor Leading Edge Technologies, Inc. (Japan)

727321 Development of polymers for non-CAR resists for EUV lithography [7273-175]

A. K. Whittaker, I. Blakey, J. Blinco, K. S. Jack, K. Lawrie, H. Liu, A. YU, The Univ. of Queensland (Australia); M. Leeson, W. Yeuh, T. Younkin, Intel Corp. (United States)

POSTER SESSION: IMMERSION LITHOGRAPHY MATERIALS AND PROCESSES

727322 CD and defect improvement challenges for immersion processes [7273-126]

K. Ehara, T. Ema, T. Yamasaki, S. Nakagawa, S. Ishitani, Toshiba Corp. (Japan); A. Morita,

J. Kim, M. Kanaoka, S. Yasuda, M. Asai, SOKUDO Co., Ltd. (Japan)

727324 Defect reduction in non-topcoat resist by selective segregation removal step [7273-129] T. Hagiwara, Renesas Technology Corp. (Japan); M. Terai, Mitsubishi Electric Corp. (Japan); T. Ishibashi, T. Miyauchi, Renesas Technology Corp. (Japan); S. Hori, Renesas Semiconductor Engineering Corp. (Japan); T. Kumada, Mitsubishi Electric Corp. (Japan); T. Kumagai,

A. Sawano, K. Doi, Tokyo Ohka Kogyo Co., Ltd. (Japan); T. Matsunobe, N. Man, H. Seki, Y. Tanahashi, Toray Research Ctr. Inc. (Japan); T. Hanawa, Renesas Technology Corp. (Japan) 
727325 Characterization of film cut position at wafer bevel for effective immersion lithography process [7273-131]

K. Matsumaro, M. Seki, T. Kato, Semiconductor Leading Edge Technologies, Inc. (Japan)

727326 High-index nanocomposite photoresist for 193-nm lithography [7273-132]

W. J. Bae, M. Trikeriotis, R. Rodriguez, M. F. Zettel, Cornell Univ. (United States); E. Piscani, Intel Corp. assignee to SEMATECH Inc. (United States); C. K. Ober, E. P. Giannelis, Cornell Univ. (United States); P. Zimmerman, Intel Corp. assignee to SEMATECH Inc. (United States)

727328 Non-topcoat process development for ArF immersion lithography [7273-134] T. Naruoka, N. Matsumura, A. Soyano, S. Kusumoto, Y. Yamaguchi, JSR Corp. (Japan); H. Arima, Y. Yoshida, K. Yoshihara, T. Shibata, Tokyo Electron Kyushu Ltd. (Japan)

727329 Backside EBR process performance with various wafer properties [7273-135] T. Goto, K. Shigemori, SOKUDO Co., Ltd. (Japan); R. Vangheluwe, ASML (Netherlands); D. Erich, Siltronic AG Corp. (Germany); M. Sanada, SOKUDO Co., Ltd. (Japan)

7273 2A High refractive index nanoparticle fluids for 193-nm immersion lithography [7273-137] M. Trikeriotis, R. Rodriguez, M. F. Zettel, A. Bakandritsos, W. J. Bae, Cornell Univ. (United States); P. Zimmerman, Intel Corp. assignee to SEMATECH Inc. (United States); C. K. Ober, E. P. Giannelis, Cornell Univ. (United States)

7273 2B Defectivity process optimization on immersion topcoat less resist stacks [7273-138] K. Shigemori, SOKUDO Co., Ltd. (Japan); S. Wang, ASML Netherlands B.V. (Netherlands); L. Tedeschi, SOKUDO USA, LLC (United States); G. Tanriseven, R. Maas, C. Verspaget, R. Marechal, A. Lammers, J. Mallmann, ASML Netherlands B.V. (Netherlands); M. Harumoto, A. Hisai, M. Asai, SOKUDO Co., Ltd. (Japan)

$72732 \mathrm{C} \quad$ Defectivity issues in topcoat-free photoresists [7273-178] K. Petrillo, R. Johnson, IBM Corp. (United States); W. Conley, FreeScale, IBM Systems \& Technology Group (United States); J. Cantone, D. Hetzer, S. Dunn, Tokyo Electron Technology Ctr. (United States); T. Winter, Tokyo Electron America, Inc. (United States); Y. van Dommelen, A. Jiang, ASML Ltd. (United States)

\section{Part Two}

\section{POSTER SESSION: LER/LWR BEHAVIOR IN RESISTS}

$72732 \mathrm{D}$ Reduction of line width and edge roughness by resist reflow process for extreme ultra-violet lithography [7273-140]

I. W. Cho, J.-M. Park, H. Kim, J.-Y. Hong, Hanyang Univ. (Korea, Republic of); S.-S. Kim, H.-K. Cho, SAMSUNG Electronics Co., Ltd. (Korea, Republic of); H.-K. Oh, Hanyang Univ. (Korea, Republic of)

$72732 \mathrm{E} \quad$ Line edge and width roughness dependency on each ingredient of extreme ultraviolet molecular resist [7273-141]

H. Kim, I. W. Cho, Hanyang Univ. (Korea, Republic of); S.-S. Kim, H.-K. Cho, SAMSUNG

Electronics Co., Ltd. (Korea, Republic of); H.-K. Oh, Hanyang Univ. (Korea, Republic of) 
$72732 \mathrm{~F} \quad$ A study of the photo acid generator material design for chemically amplified photoresists [7273-143]

Y. Utsumi, T. Seshimo, Y. Komuro, A. Kawave, K. Ishiduka, K. Matsuzawa, H. Hada,

J. Onodera, Tokyo Ohka Kogyo Co., Ltd. (Japan)

72732 S Stochastic modeling in lithography: autocorrelation behavior of catalytic reaction-diffusion systems [7273-145]

C. A. Mack, Consultant (United States)

$72732 \mathrm{H} \quad$ Important challenges for line-width-roughness reduction [7273-146]

H. Yaegashi, Tokyo Electron Ltd. (Japan); M. Kushibiki, E. Nishimura, Tokyo Electron AT Ltd.

(Japan); S. Shimura, F. Iwao, T. Kawasaki, Tokyo Electron Kyushu Ltd. (Japan); K. Hasebe, H. Murakami, Tokyo Electron Tohoku Ltd. (Japan); A. Hara, K. Yabe, Tokyo Electron Ltd.

(Japan)

$727321 \quad$ Stochastic modeling in lithography: autocorrelation behavior of catalytic reaction-diffusion systems [7273-145]

C. A. Mack, Consultant (United States)

$72732 \mathrm{~J}$ Line edge roughness transfer during plasma etching: modeling approaches and comparison with experimental results [7273-177]

V. Constantoudis, G. Kokkoris, P. Xydi, E. Gogolides, Institute of Microelectronics, NCSR Demokritos (Greece); E. Pargon, M. Martin, Lab. des Technologies de la Microélectronique, CNRS (France)

\section{POSTER SESSION: MOLECULAR RESISTS}

7273 2K Image reversal trilayer process using standard positive photoresist [7273-148]

D. J. Abdallah, J. Sagan, AZ Electronic Materials (United States); K. Kurosawa, J. Li, Y. Takano, Y. Shimizu, N. Shinde, T. Nagahara, T. Ishikawa, AZ Electronic Materials (Japan);

R. R. Dammel, AZ Electronic Materials (United States)

7273 2L Quantitative measurement of the molecular-mass distribution in calix[4]resorcinarene molecular glass resists by mass spectrometry [7273-149]

W. E. Wallace, K. M. Flynn, C. M. Guttman, D. L. VanderHart, V. M. Prabhu, National Institute of Standards and Technology (United States); A. De Silva, N. M. Felix, C. K. Ober, Cornell Univ. (United States)

7273 2M Adamantane-based molecular glass resist for 193-nm lithography [7273-151]

S. Tanaka, M. Murakami, K. Fukushima, N. Kawano, Y. Uenoyama, K. Ito, H. Ohno,

N. Matsumoto, Idemitsu Kosan Co., Ltd. (Japan)

$72732 \mathrm{~N} \quad$ Molecular glass resists for next-generation lithography [7273-152]

M. Krysak, A. De Silva, J. Sha, J.-K. Lee, C. K. Ober, Cornell Univ. (United States)

727320 Reworkable spin-on trilayer materials: optimization of rework process and solutions for manufacturability [7273-153]

R. Zhang, A. G. Timko, J. Zook, Y. Wei, L. Pylneva, Y. Yi, C. Li, H. WU, D. Rahman, D. S. McKenzie, C. Anyadiegwu, P. Lu, M. Neisser, R. R. Dammel, R. Bradbury, T. Lee, AZ Electronic Materials (United States) 
$72732 \mathrm{Q}$ Development of new phenylcalix[4]resorcinarene: its application to positive-tone molecular resist for EB and EUV lithography [7273-155]

M. Echigo, D. Oguro, Mitsubishi Gas Chemical Co., Inc. (Japan)

$72732 R \quad$ Development of novel positive-tone resists for EUVL [7273-156]

T. Owada, A. Yomogita, T. Kashiwamura, T. Kusaba, S. Miyamoto, T. Takeya, Idemitsu Kosan Co., Ltd. (Japan)

$72732 \mathrm{~T} \quad$ Molecular glass resists developable in supercritical carbon dioxide for $193 \mathrm{~nm}$ lithography [7273-158]

J. Sha, J. Lee, C. K. Ober, Cornell Univ. (United States)

$72732 \mathrm{U} \quad$ Molecular resists based on calix[4]resorcinarene derivatives for EB lithography [7273-159]

K. Okuyama, Dai Nippon Printing Co., Ltd. (Japan)

7273 2V Practical implementation of immersion resist materials [7273-160]

H. Khorram, Nikon Precision Inc. (United States); K. Nakanob, T. Fujiwara, Y. Iriuchijima, Y. Ishii, N. Sagawa, T. Kawakubo, Nikon Corp. (Japan); S. Nagaoka, Nikon Precision Inc. (United States)

$72732 \mathrm{~W} \quad$ Analysis of molecular resist distribution in a resist film by using $\mathrm{x}$-ray reflectivity [7273-161] J. Kim, J. Lee, D. Kim, J. Kim, Dongjin Semichem Co. Ltd. (Korea, Republic of); S. Ahn, W. Zin, Pohang Univ. of Science and Technology (Korea, Republic of)

\section{POSTER SESSION: RESIST FUNDAMENTALS}

$72732 X \quad$ Theoretical analysis of energy degradation of electrons in the resists [7273-162] M. Toriumi, Lab. for Interdisciplinary Science and Technology (Japan)

$72732 Y \quad$ Theoretical analysis of development behavior of resist measured by QCM [7273-163] M. Toriumi, Lab. for Interdisciplinary Science and Technology (Japan)

$72732 Z$ Temperature and critical dimension variation in a single wafer on hot plate due to non-uniform heat source [7273-164]

B. Kim, J.-M. Park, H. Kim, I. An, S.-W. Park, H.-K. Oh, Hanyang Univ. (Korea, Republic of)

727330 Decomposition analysis of molecular resists to further CD control [7273-165]

D. Shiono, H. Hada, T. Hirayama, J. Onodera, Tokyo Ohka Kogyo Co., Ltd. (Japan);

T. Watanabe, H. Kinoshita, Univ. of Hyogo (Japan)

727331 Diffusion of acid and amine at resist/BARC interface [7273-166]

M. Shirai, N. Majima, H. Okamura, Osaka Prefecture Univ. (Japan); Y. Hiroi, S. Kimura, Y. Nakajima, Nissan Chemical Industries, Ltd. (Japan)

727332 EUV resist requirements: absorbance and acid yield [7273-167]

R. Gronheid, IMEC (Belgium); C. Fonseca, Tokyo Electron America, Inc. (United States); M. J. Leeson, Intel Corp. assignee to Intel (Belgium); J. R. Adams, IMEC (Belgium) and The Univ. of Texas at Austin (United States); J. R. Strahan, C. G. Willson, The Univ. of Texas at Austin (United States); B. W. Smith, Rochester Institute of Technology (United States) 
727333 Study of residue type defect formation mechanism and the effect of advanced defect reduction (ADR) rinse process [7273-168]

H. Arima, Y. Yoshida, K. Yoshihara, T. Shibata, Tokyo Electron Kyushu Ltd. (Japan); Y. Kushida, H. Nakagawa, Y. Nishimura, Y. Yamaguchi, JSR Corp. (Japan)

727334 Understanding pattern collapse in high-resolution lithography: impact of feature width on critical stress [7273-180]

D. E. Noga, R. A. Lawson, C.-T. Lee, L. M. Tolbert, C. L. Henderson, Georgia Institute of Technology (United States)

\section{POSTER SESSION: SIMULATION OF RESIST PROCESSES}

727335 Characteristic three-dimensional structure of resist's distribution after drying a resist solution coated on a flat substrate: analysis using the extended dynamical model of the drying process [7273-170]

H. Kagami, Nagoya College (Japan)

727336 Polymer dissolution model: an energy adaptation of the critical ionization theory [7273-172] S. Chauhan, The Univ. of Texas at Austin (United States); M. Somervell, S. Scheer, Tokyo Electron America, Inc. (United States); C. A. Mack, Lithoguru.com (United States);

R. T. Bonnecaze, C. G. Willson, The Univ. of Texas at Austin (United States)

727337 Meso-scale simulation of the polymer dynamics in the formation process of line-edge roughness [7273-173]

H. Morita, National Institute of Advanced Industrial Science and Technology (Japan);

M. Doi, Univ. of Tokyo (Japan)

IMMERSION LITHOGRAPHY MATERIALS AND PROCESSES

727338 Improvements in process performance for immersion technology high volume manufacturing [7273-13]

K. Nafus, T. Shimoaoki, M. Enomoto, H. Shite, T. Otsuka, H. Kosugi, T. Shibata, Tokyo Electron Kyushu Ltd. (Japan); J. Mallmann, R. Maas, C. Verspaget, E. van der Heijden, E. van Setten, J. Finders, S. Wang, N. Boudou, C. Zoldesi, ASML Netherlands B.V. (Netherlands)

727339 Advanced immersion contact hole patterning for sub 40nm memory applications: a fundamental resist study [7273-14]

Y.-K. Jang, J.-Y. Yoon, S.-Y. Lee, K.-S. Yoon, S.-H. Oh, S.-W. Choi, W.-S. Han, SAMSUNG Electronics Co., Ltd. (Korea, Republic of); S. Kang, T. Penniman, D.-S. Kim, D. W. Chung, S.-S. Cho, C. B. XU, G. Barclay, Rohm and Haas Electronic Materials (United States)

7273 3A Resist process control for 32-nm logic node and beyond with NA > 1.30 immersion exposure tool [7273-15]

S. Nagahara, NEC Electronics Corp. (Japan); K. Takahata, S. Nakagawa, Toshiba Corp. (Japan); T. Murakami, K. Takeda, S. Nakamura, M. Ueki, NEC Electronics Corp. (Japan); M. Satake, T. Ema, H. Fujise, H. Yonemitsu, Y. Seino, S. Nakagawa, M. Asano, Y. Kitamura, Toshiba Corp. (Japan); T. Uchiyama, NEC Electronics Corp. (Japan); S. Mimotogi, Toshiba Corp. (Japan); M. Tominaga, NEC Electronics Corp. (Japan) 
7273 3B Feasibility study of non-topcoat resist for $22 \mathrm{~nm}$ node devices [7273-16]

K. Sho, H. Kato, K. Kobayashi, K. lida, T. Ori, D. Muto, T. Azuma, S. Ito, Toshiba Corp. (Japan); T. Fujiwara, Y. Ishii, Nikon Corp. (Japan); Y. Nishimura, T. Kawakami, M. Shima, JSR Corp.

(Japan)

\section{MOLECULAR RESISTS}

7273 3C Single component molecular resists containing bound photoacid generator functionality [7273-17]

R. A. Lawson, L. M. Tolbert, C. L. Henderson, Georgia Institute of Technology (United States)

7273 3D Low activation energy fullerene molecular resist [7273-18]

J. Manayam, M. Manickam, J. A. Preece, R. E. Palmer, A. P. G. Robinson, The Univ. of

Birmingham (United Kingdom)

7273 3E Negative-tone molecular resists based on cationic polymerization [7273-19]

R. A. Lawson, L. M. Tolbert, Georgia Institute of Technology (United States); T. R. Younkin, Intel

Corp. (United States); C. L. Henderson, Georgia Institute of Technology (United States)

\section{NOVEL MATERIALS, PROCESSES, AND APPLICATIONS I}

$72733 \mathrm{G}$ Fluorinated polymethacrylates as highly sensitive non-chemically amplified e-beam resists [7273-21]

J. R. Strahan, The Univ. of Texas at Austin (United States); J. R. Adams, The Univ. of Texas at Austin (United States) and IMEC Vwz (Belgium); W. Jen, The Univ. of Texas at Austin (United States); A. Vanleenhove, NXP Semiconductors (Belgium); C. C. Neikirk, T. Rochelle, The Univ. of Texas at Austin (United States); R. Gronheid, IMEC (Belgium); C. G. Willson, The Univ. of Texas at Austin (United States)

$72733 \mathrm{H} \quad$ Hybrid resist systems based on $\alpha$-substituted acrylate copolymers [7273-22] H. Ito, L. K. Sundberg, L. Bozano, E. M. Lofano, IBM Almaden Research Ctr. (United States); K. Yamanaka, Y. Terui, M. Fujiwara, Central Glass Co., Ltd. (Japan)

$727331 \quad$ Sub-20 $\mathrm{nm}$ trench patterning with a hybrid chemical shrink and SAFIER process [7273-23] Y. Chen, X. XU, H. Chen, L. Miao, P. Blanco, M. Cai, C. S. Ngai, Applied Materials, Inc. (United States)

\section{ARCS AND MULTILAYER PROCESSES}

7273 3L Progress towards production worthy developable BARCs (DBARCs) [7273-27]

J. Cameron, J. Amara, G. Prokopowicz, J. W. Sung, D. Valeri, A. Ware, K. O'Shea, Rohm and Haas Electronic Materials LLC (United States); Y. Yamamoto, T. Kurihara, Rohm and Haas Electronic Materials K.K. (Japan); L. Vyklicky, W. Huang, I. Popova, P. R. Varanasi, IBM Corp. (United States)

7273 3M High-Si content BARC for dual-BARC systems such as trilayer patterning [7273-28] J. Kennedy, S. Xie, Z. Wu, R. Katsanes, K. Flanigan, Honeywell Electronic Materials (United States); K. Lee, Honeywell Taiwan Ltd. (Taiwan); M. Slezak, Z. Liu, S. Lin, JSR Micro, Inc. (United States) 
$72733 \mathrm{~N}$ Incorporating organosilanes into EUV photoresists: diphenyltrimethylsilylmethylsulfonium triflate as a new PAG [7273-29]

S. Sharma, JSR Micro, Inc. (United States); Y. Ogata, JSR Micro, Inc. (United States) and LBNL Molecular Foundry (United States); C. Tung, LBNL Molecular Foundry (United States);

J. M. Blackwell, LBNL Molecular Foundry (United States) and Intel Corp. (United States);

T. R. Younkin, Intel Corp. (United States); Y. Hishiro, JSR Micro, Inc. (United States);

J. S. Figueroa, A. L. Rheingold, Univ. of California, San Diego (United States)

727330 Main chain decomposible star shaped polymer for EUV resist [7273-30]

J. Iwashita, T. Mimura, T. Hirayama, T. Iwai, Tokyo Ohka Kogyo Co., Ltd. (Japan)

$72733 \mathrm{P} \quad$ Resist material design to improve sensitivity in EUV lithography [7273-31]

H. Tsubaki, T. Tsuchihashi, K. Yamashita, T. Tsuchimura, FUJIFILM Co., Ltd. (Japan)

$72733 Q \quad$ Lithographic evaluation and chemical modeling of acid amplifiers used in EUV photoresists [7273-32]

R. Brainard, C. Higgins, S. Kruger, S. Revuru, B. Cardineau, Univ. at Albany (United States);

S. Gibbons, D. Freedman, State Univ. of New York at New Paltz (United States); H. Solak, Paul Scherrer Institute (Switzerland); W. Yueh, T. Younkin, Intel Corp. (United States)

$72733 R \quad$ Aryl sulfonates as neutral photoacid generators (PAGs) for EUV lithography [7273-33]

R. Sulc, LBNL Molecular Foundry (United States); J. M. Blackwell, LBNL Molecular Foundry (United States) and Intel Corp. (United States); T. R. Younkin, E. S. Putna, Intel Corp. (United States); K. Esswein, A. G. DiPasquale, Univ. of California, Berkeley (United States);

R. Callahan, FUJIFILM Electronic Materials U.S.A., Inc. (United States); H. Tsubaki,

T. Tsuchihashi, FUJIFILM Corp. (Japan)

727335 Effect of resist polymer molecular weight on EUV lithography [7273-34]

H. Tsubaki, K. Yamashita, H. Takahashi, FUJIFILM Co., Ltd (Japan); D. Kawamura, T. Itani, Semiconductor Leading Edge Technologies, Inc. (Japan)

7273 3т Correlation of EUV resist performance metrics in micro-exposure and full-field EUV projection tools [7273-35]

T. I. Wallow, GlobalFoundries (United States); B. Pierson, ASML (United States); H. Mizuno, Toshiba America Electronic Components, Inc. (United States); A. Fumar-Pici, ASML (United States); K. Petrillo, IBM Corp. (United States); C. N. Anderson, Univ. of California, Berkeley (United States); P. P. Naulleau, Lawrence Berkeley National Lab. (United States);

S. G. Hansen, ASML (United States); Y. Deng, GlobalFoundries (United States);

K. van Ingen Schenau, ASML (Netherlands); C.-S. Koay, IBM Corp. (United States); L. Ohara, S.-I. Han, R. Watso, ASML (United States); L. Huli, Univ. at Albany (United States); M. Burkhardt, IBM Corp. (United States); O. Wood, GlobalFoundries (United States); J. Mallmann, B. Kessels, ASML (Netherlands); R. Routh, K. Cummings, ASML (United States)

RESIST FUNDAMENTALS

$72733 \mathrm{U} \quad$ Characterization of the photoacid diffusion length [7273-36]

S. Kang, V. M. Prabhu, W WU, E. K. Lin, National Institute of Standards and Technology (United States); K.-W. Choi, M. Chandhok, T. R. Younkin, W. Yueh, Intel Corp. (United States) 
$72733 \mathrm{~V}$ Elucidating the physiochemical and lithographic behavior of ultra-thin photoresist films [7273-37]

A. Sundaramoorthi, Georgia Institute of Technology (United States); T. R. Younkin, Intel Corp. (United States); C. L. Henderson, Georgia Institute of Technology (United States)

7273 3X Non-CA resists for $193 \mathrm{~nm}$ immersion lithography: effects of chemical structure on sensitivity [7273-39]

I. Blakey, L. Chen, Y.-K. Goh, K. Lawrie, Y Chuang, The Univ. of Queensland (Australia);

E. Piscani, P. A. Zimmerman, SEMATECH, Inc. (United States); A. K. Whittaker, The Univ. of

Queensland (Australia)

$72733 Z$ Quantitative measurement of resist outgassing during exposure [7273-41]

N. Maxim, ASML Netherlands B.V. (Netherlands); F. A. Houle, IBM Almaden Research Ctr. (United States); J. Huijbregtse, ASML Netherlands B.V. (Netherlands); V. R. Deline, H. Truong, IBM Almaden Research Ctr. (United States); W. van Schaik, ASML Netherlands B.V. (Netherlands)

\section{SIMULATION OF RESIST PROCESSES}

727340 Simulation of optical lithography in the presence of topography and spin-coated films [7273-42]

S. A. Robertson, KLA-Tencor Corp. (United States); M. T. Reilly, Rohm and Haas Electronic Materials LLC (United States); T. Graves, J. J. Biafore, M. D. Smith, KLA-Tencor Corp. (United States); D. Perret, Rohm and Haas Electronic Materials (France); V. Ivin, S. Potashov, M. Silakov, N. Elistratov, Mirantis (Russian Federation)

$727341 \quad$ Mesoscale kinetic Monte Carlo simulations of molecular resists: the effect of PAG homogeneity on resolution, LER, and sensitivity [7273-44]

R. A. Lawson, C. L. Henderson, Georgia Institute of Technology (United States)

727342 Calibration of physical resist models: methods, usability, and predictive power [7273-45]

U. K. Klostermann, T. Mülders, D. Ponomarenco, T. Schmöller, Synopsys GmbH (Germany); J. Van de Kerkhove, P. De Bisschop, IMEC (Belgium)

727343 Statistical simulation of resist at EUV and ArF [7273-46]

J. J. Biafore, M. D. Smith, KLA-Tencor Corp. (United States); C. A. Mack, Lithoguru.com (United States); J. W. Thackeray, Rohm and Haas Electronic Materials (United States); R. Gronheid, IMEC (Belgium); S. A. Robertson, T. Graves, D. Blankenship, KLA-Tencor Corp. (United States)

727344 Position shift analysis in resist reflow process for sub-50-nm contact hole [7273-47] J.-H. You, J. Park, J. -M Park, H. Jeong, H.-K. Oh, Hanyang Univ. (Korea, Republic of)

\section{LER/LWR BEHAVIOR IN RESISTS}

727345 Resist roughness bi-modality as revealed by two-dimensional FFT 2D analysis [7273-48] Y. Gotkis, L. Baranov, KLA-Tencor Corp. (United States); T. H. Fedynyshyn, S. Cann, MIT Lincoln Lab. (United States) 
727346 Reducing LER using a grazing incidence ion beam [7273-49]

C. R. M. Struck, R. Raju, M. J. Neumann, D. N. Ruzic, Univ. of Illinois at Urbana-Champaign (United States)

727347 Resist fundamentals for resolution, LER, and sensitivity (RLS) performance tradeoffs and their relation to micro-bridging defects [7273-50]

B. Rathsack, Tokyo Electron America, Inc. (United States); K. Nafus, S. Hatakeyama, Y. Kuwahara, J. Kitano, Tokyo Electron Kyushu Ltd. (Japan); R. Gronheid, A. Vaglio Pret, IMEC (Belgium)

727349 PAG segregation during exposure affecting innate material roughness [7273-52] T. H. Fedynyshyn, D. K. Astolfi, A. Cabral, S. Cann, I. Pottebaum, MIT Lincoln Lab. (United States); J. M. Roberts, Intel Corp. (United States)

\section{NOVEL MATERIALS, PROCESSES, AND APPLICATIONS II}

7273 4A Chalcogenide glass thin film resists for grayscale lithography [7273-53]

A. Kovalskiy, J. Cech, C. L. Tan, W. R. Heffner, E. Miller, Lehigh Univ. (United States);

C. M. Waits, M. Dubey, W. Churaman, Army Research Lab. (United States); M. Vlcek, Univ. of Pardubice (Czech Republic); H. Jain, Lehigh Univ. (United States)

7273 4B Defect reduction by using point-of-use filtration in a new coater/developer [7273-54] T. Umeda, S. Tsuzuki, T. Numaguchi, Nihon Pall Ltd. (Japan)

7273 4C CDU improvement with wafer warpage control oven for high-volume manufacturing [7273-55]

T. Tomita, H. Weichert, Tokyo Electron Europe Ltd. (Germany); S. Hornig, S. Trepte, Qimonda Dresden GmbH \& Co. KG (Germany); H. Shite, R. Uemura, J. Kitano, Tokyo Electron Kyushu Ltd. (Japan)

7273 4D Gap-fill type HSQ/ZEP520A bilayer resist process-(III): optimal process window for HSQ air-tip formation [7273-56]

W.-S. Chen, M.-J. Tsai, Industrial Technology Research Institute (Taiwan)

$72734 \mathrm{E} \quad$ Feasibility studies of coating method for planarization process [7273-57]

K. Matsunaga, T. Oori, H. Kato, E. Shiobara, Toshiba Corp. (Japan); M. Muramatsu, M. Iwashita, T. Kitano, Tokyo Electron Kyushu Ltd. (Japan); Y. Horiguchi, T. Ohashi, S. Takei, Nissan Chemical Industries, Ltd. (Japan); S. Ito, Toshiba Corp. (Japan)

$72734 \mathrm{~F}$ Contact analysis studies of an ESCAP resist with $\mathrm{scCO}_{2}$ compatible additives [7273-58] A. Rastogi, Cornell Univ. (United States); G. N. Toepperwein, Univ. of Wisconsin, Madison (United States); M. Tanaka, Cornell Univ. (United States); R. A. Riggleman, J. J. de Pablo, Univ. of Wisconsin, Madison (United States); C. K. Ober, Cornell Univ. (United States) 


\title{
Symposium Committees
}

\author{
Symposium Chair
}

Christopher J. Progler, Photronics, Inc. (United States)

Symposium Cochair

Donis G. Flagello, Nikon Corporation of America (United States)

Technical Organizing Committees

Executive Committee

John A. Allgair, SEMATECH Inc. (United States) and Advanced Micro Devices, Inc. (United States)

Mircea V. Dusa, ASML MaskTools, Inc. (United States)

Donis G. Flagello, Nikon Corporation of America (United States)

Clifford L. Henderson, Georgia Institute of Technology (United States)

Bruno La Fontaine, Advanced Micro Devices, Inc. (United States)

Harry J. Levinson, Advanced Micro Devices, Inc. (United States)

Christopher J. Progler, Photronics, Inc. (United States)

Christopher J. Raymond, Nanometrics, Inc. (United States)

Michael L. Rieger, Synopsys, Inc. (United States)

Franklin M. Schellenberg, Mentor Graphics Corporation (United States)

Vivek K. Singh, Intel Corporation (United States)

Advisory Committee

Robert D. Allen, IBM Almaden Research Center (United States)

William H. Arnold, ASML US Inc. (United States)

Timothy A. Brunner, IBM Thomas J. Watson Research Center (United States)

Ralph R. Dammel, AZ Electronic Materials USA Corporation (United States)

Roxann L. Engelstad, University of Wisconsin, Madison (United States)

Roderick R. Kunz, MIT Lincoln Laboratory (United States)

Harry J. Levinson, Advanced Micro Devices, Inc. (United States)

Burn Lin, Taiwan Semiconductor Manufacturing Company, Ltd. (Taiwan)

Chris A. Mack, LithoGuru.com (United States)

Victor Pol, Freescale Semiconductor, Inc. (United States) 
Michael T. Postek, National Institute of Standards and Technology (United States)

Luc Van den Hove, IMEC vzW (Belgium)

C. Grant Willson, The University of Texas at Austin (United States) Anthony Yen, Taiwan Semiconductor Manufacturing Company, Ltd. (Taiwan) 


\title{
Conference Committee
}

\author{
Conference Chair
}

Clifford L. Henderson, Georgia Institute of Technology (United States)

Conference Cochair

Robert D. Allen, IBM Almaden Research Center (United States)

Program Committee

George G. Barclay, Rohm and Haas Electronic Materials (United States)

Sean D. Burns, IBM Thomas J. Watson Research Center (United States)

Ralph R. Dammel, AZ Electronic Materials (United States)

Douglas J. Guerrero, Brewer Science, Inc. (United States)

Christoph K. Hohle, Qimonda Dresden GmbH \& Co. OHG (Germany)

Qinghuang Lin, IBM Thomas J. Watson Research Center (United States)

Nobuyuki N. Matsuzawa, Sony Atsugi Technology Center (Japan)

Dah-Chung Owe-Yang, Shin-Etsu MicroSi, Inc. (United States)

Adam R. Pawloski, Affymetrix, Inc. (United States)

Vivek M. Prabhu, National Institute of Standards and Technology (United States)

E. Steve Putna, Intel Corporation (United States)

Mark H. Somervell, Tokyo Electron America, Inc. (United States)

Gregory M. Wallraff, IBM Almaden Research Center (United States)

\section{Session Chairs}

Welcome and Announcements

Clifford L. Henderson, Georgia Institute of Technology (United States)

Robert D. Allen, IBM Almaden Research Center (United States)

1 Keynote Session

Robert D. Allen, IBM Almaden Research Center (United States)

2 Double Patterning and Double Exposure I

Robert D. Allen, IBM Almaden Research Center (United States)

Vivek M. Prabhu, National Institute of Standards and Technology (United States)

3 Double Patterning and Double Exposure II

George G. Barclay, Rohm and Haas Electronic Materials (United States)

Sean D. Burns, IBM Thomas J. Watson Research Center (United States) 
$4 \quad$ Immersion Lithography Materials and Processes

George G. Barclay, Rohm and Haas Electronic Materials (United States)

Dah-Chung Owe-Yang, Shin-Ełsu MicroSi, Inc. (United States)

5 Molecular Resists

Clifford L. Henderson, Georgia Institute of Technology (United States)

Mark H. Somervell, Tokyo Electron America, Inc. (United States)

6 Novel Materials, Processes, and Applications I

Adam R. Pawloski, Affymetrix, Inc. (United States)

Vivek M. Prabhu, National Institute of Standards and Technology (United States)

$7 \quad$ ARCs and Multilayer Processes

Ralph R. Dammel, AZ Electronic Materials (United States)

Douglas J. Guerrero, Brewer Science, Inc. (United States)

8 EUV Resist Materials and Processes

E. Steve Putna, Intel Corporation (United States)

Nobuyuki N. Matsuzawa, Sony Atsugi Technology Center (Japan)

9 Resist Fundamentals

Gregory M. Wallraff, IBM Almaden Research Center (United States)

Ralph R. Dammel, AZ Electronic Materials (United States)

10 Simulation of Resist Processes

Adam R. Pawloski, Affymetrix, Inc. (United States)

Vivek M. Prabhu, National Institute of Standards and Technology (United States)

11 LER/LWR Behavior in Resists

Qinghuang Lin, IBM Thomas J. Watson Research Center (United States)

Mark H. Somervell, Tokyo Electron America, Inc. (United States)

12 Novel Materials, Processes, and Applications II

E. Steve Putna, Intel Corporation (United States)

Mark H. Somervell, Tokyo Electron America, Inc. (United States) 